\title{
Pharmacogenetics in type 2 diabetes: precision medicine or discovery tool?
}

\author{
Jose C. Florez ${ }^{1,2,3,4,5}$
}

Received: 10 October 2016 / Accepted: 25 January 2017 / Published online: 10 March 2017

(C) Springer-Verlag Berlin Heidelberg 2017

\begin{abstract}
In recent years, technological and analytical advances have led to an explosion in the discovery of genetic loci associated with type 2 diabetes. However, their ability to improve prediction of disease outcomes beyond standard clinical risk factors has been limited. On the other hand, genetic effects on drug response may be stronger than those commonly seen for disease incidence. Pharmacogenetic findings may aid in identifying new drug targets, elucidate pathophysiology, unravel disease heterogeneity, help prioritise specific genes in regions of genetic association, and contribute to personalised or precision treatment. In diabetes, precedent for the successful application of pharmacogenetic concepts exists in its monogenic subtypes, such as MODY or neonatal diabetes. Whether similar insights will emerge for the much more common entity of type 2 diabetes remains to be seen. As genetic approaches advance, the progressive deployment of candidate gene, large-scale genotyping and genome-wide association studies has begun to produce suggestive results
\end{abstract}

Electronic supplementary material The online version of this article (doi:10.1007/s00125-017-4227-1) contains a slideset of the figures for download, which is available to authorised users.

Jose C. Florez

jcflorez@partners.org

1 Diabetes Unit, Massachusetts General Hospital, Boston, MA, USA

2 Center for Genomic Medicine, Massachusetts General Hospital, Simches Research Building-CPZN 5.250, 185 Cambridge Street, Boston, MA 02114, USA

3 Metabolism Program, Broad Institute, Cambridge, MA, USA

4 Program in Medical and Population Genetics, Broad Institute, Cambridge, MA, USA

5 Department of Medicine, Harvard Medical School, Boston, MA, USA that may transform clinical practice. However, many barriers to the translation of diabetes pharmacogenetic discoveries to the clinic still remain. This perspective offers a contemporary overview of the field with a focus on sulfonylureas and metformin, identifies the major uses of pharmacogenetics, and highlights potential limitations and future directions.

Keywords Genome-wide association studies · Metformin · Pharmacogenetics $\cdot$ Review $\cdot$ Single nucleotide polymorphisms $\cdot$ Sulfonylureas $\cdot$ TCF7L2 - Type 2 diabetes

$\begin{array}{ll}\text { Abbreviations } \\ \text { AMPK } & \text { AMP-activated protein kinase } \\ \text { DPP } & \text { Diabetes Prevention Program } \\ \text { GoDARTS } & \begin{array}{l}\text { Genetics of Diabetes and Audit Research } \\ \text { Tayside Study }\end{array} \\ \text { GWAS } & \text { Genome-wide association studies } \\ \text { OCT1 } & \text { Organic cation transporter 1 } \\ \text { SNP } & \text { Single nucleotide polymorphism } \\ \text { TCF7L2 } & \text { Transcription factor 7-like 2 }\end{array}$

\section{Introduction}

Pharmacogenetics is the discipline that studies the interaction between genetic variation and drug exposure on modulating therapeutic response. To the extent that a drug is more or less effective in a specific genetic context, pharmacogenetic information may be clinically useful, for example, in medication selection or dose titration. Conversely, a drug that attenuates the pathological consequences of a genetic defect may ameliorate the clinical impact of the mutation it targets (the reciprocal view of the same statistical relationship). As a corollary, clinical response to a drug for which the mechanism 
of action in carriers of a specific variant is known can also be informative with regard to the function of the gene affected by the variant [1].

In recent years, pharmacogenetic studies have been greatly facilitated by the growing catalogue of human genetic variation, as well as parallel technological advances that enable the high-throughput generation of genetic data in large populations. These have taken the form of targeted explorations of known genetic variants (e.g. in genes that encode proteins known to affect the pharmacokinetic or pharmacodynamic properties of a given drug), or of agnostic searches that query the entire genome to search for variants that influence drug response. Proof-of-concept approaches have been immensely successful in oncology, where design drugs have targeted unique somatic mutations that occur in the patient's tumour. Whether similar approaches are likely to succeed for germline (inherited) genetic variation, particularly for complex polygenic diseases such as type 2 diabetes, is the topic of this review.

\section{Goals of pharmacogenetic studies}

Because of the richness inherent to pharmacogenetic data and the diverse perspectives from which they can be interpreted, it is crucial to clarify the purpose of each research exercise upfront. Study design, analysis of results and biological inference may vary depending on the goals and hypotheses that guide a given experiment. Pharmacogenetic studies may pursue one or several major objectives:

1. Patient stratification Genetic information may be used to define subgroups of individuals more or less likely to experience clinical response to the drug in question, or to develop side effects. This is the hope articulated by precision medicine, which aspires to administer the right medication to the right patient at the right time.

2. Target identification Genetic studies may help with the discovery of novel drug targets that illuminate mechanisms of action and open new therapeutic avenues, particularly if their scope is genome-wide and their approach is unbiased.

3. Functional characterisation Drugs can also be used to perturb the human organism in vivo. If such a perturbation generates a physiological response that differs depending on genetic variation at a specific locus, insights may be gained with respect to the mode of action of the gene product, or the cell type in which it is expressed. In this manner, pharmacogenetic experiments can offer proof-of-concept evidence that implicates a given gene in a molecular pathway and strengthens the evidence suggesting that an observed association is causal.
Multiple reports of associations between genetic variants and drug response for any one of the 12 drug classes currently approved for the treatment of type 2 diabetes have been published [2-4], although the degree of confidence in the reported results varies greatly. To a large extent, this is due to the limited sample sizes currently available for studies into non-generic drugs, as most such studies are the province of individual pharmaceutical companies, and firm executives may be wary of limiting the marketability of a proprietary compound by attaching a genetic indication. Similarly, successful pharmacogenetic approaches for target identification in the pursuit of drug discovery may remain private until the new drug is brought to market. Thus, academic investigators are often limited to working with generic drugs, and perturbation trials that use novel agents are typically restricted to sample sizes that are too small to generate robust statistical confidence. Nevertheless, the following sections offer examples that illustrate how these three distinct approaches have been applied in type 2 diabetes.

\section{Patient stratification: sulfonylureas in monogenic and polygenic diabetes}

\section{Neonatal diabetes: mutations in $A B C C 8$ or $K C N J 11$}

The ability to clearly separate patients into responders and non-responders is largely predicated on the magnitude of the genetic effect on drug bioavailability or efficacy (pharmacokinetics and pharmacodynamics), or on the clinical phenotype the drug is meant to correct. Therefore, monogenic syndromes (where a single mutation has a strong effect on phenotype) constitute the natural theatre in which to attempt clinical validation. In diabetes, the cleanest example is provided by neonatal diabetes caused by activating mutations in the genes that encode the sulfonylurea receptor $(A B C C 8)$ or its associated ATP-dependent potassium channel (KCNJ11), which lie adjacent to each other in chromosome 11. Constitutively activating mutations that lead to hyperactivity of this complex impair the ability of beta cells to depolarise in response to a glucose stimulus, hindering insulin secretion and causing insulin insufficiency $[5,6]$. The resulting hyperglycaemia is typically detected in the first year of life and must be distinguished from the autoimmune destruction of pancreatic beta cells that occurs in type 1 diabetes. Though very early onset ( $<6$ months) and the absence of autoantibodies favour the diagnosis of neonatal diabetes, genetic confirmation is typically required [7]. In many of these cases, the genetic defect can be overcome by high doses of the very same medication class (sulfonylureas) that targets the sulfonylurea receptor/potassium channel complex [6, 8]. Patients with documented mutations in the relevant genes can be safely transitioned to an oral regimen and shed multiple 
daily insulin injections; their sustained improvement in glycaemic response demonstrates that the use of pharmacogenetic information in patient care can improve not only the quality of care but also the quality of life.

\section{Type 2 diabetes: polymorphisms in $K C N J 11$ and $A B C C 8$}

Other variants at the KCNJ11-ABCC8 gene locus that have weaker effects increase risk of common type 2 diabetes. The $\mathrm{T}$ allele at the common missense single nucleotide polymorphism (SNP) rs5219 in KCNJ11, by which a glutamate becomes a lysine at position 23 (E23K), raises the odds of type 2 diabetes by $\sim 15 \%$ [9]. Carriers also demonstrate diminished insulin secretion in response to a glucose challenge, though not to the extent seen in neonatal diabetes [10]. However, the E23K polymorphism in KCNJ11 is in near-complete linkage disequilibrium with another missense polymorphism in the adjacent gene $A B C C 8$ (rs757110), by which a serine becomes an alanine at position 1369 of the sulfonylurea receptor (S1369A). As a result, virtually every carrier of the risk $\mathrm{K}$ allele at $K C N J 11$ carries the A allele at $A B C C 8$, making it impossible to distinguish the source of the association signal on purely genetic grounds [10]. To discriminate between the two variants experimental manipulation is required, as human genetic evolutionary history has not separated them via recombination. Potassium currents can be measured in vitro by patch-clamp techniques applied to human cells transfected with the recombinant construct; the activation of the potassium current after gliclazide administration is diminished in cells transfected with the A1369/K23 haplotype, reflecting the human genetic defect [11]. When the constructs are scrambled to generate the four possible combinations at the two sites, the A1369/K23 and A1369/E23 constructs display reduced conductance when compared with the S1369/K23 and S1369/E23 constructs, demonstrating that, for gliclazide response, the likely culprit variant is A1369 in $A B C C 8$, and not $\mathrm{K} 23$ in KCNJ11.

To take this finding into human populations, Chinese investigators tested two cohorts of participants with type 2 diabetes of relatively recent onset [12]. All participants responded to gliclazide treatment by improvements in fasting glucose and $\mathrm{HbA}_{1 \mathrm{c}}$ levels after 2 months, but carriers of the A1369 polymorphism had a significantly stronger response, with an additional improvement in fasting glucose of $\sim 0.67 \mathrm{mmol} / 1$ when compared with S1369 carriers, a finding that is clinically significant. This result has been replicated using $\mathrm{HbA}_{1 \mathrm{c}}$ as the response metric and KCNJ11 E23K as the genetic marker [13]. It recapitulates the observation in neonatal diabetes and suggests that sulfonylureas can be used to overcome genetic defects in their molecular target. A small study did not reveal higher incidence of hypoglycaemia caused by this heightened sensitivity [14]; whether differences in medication potency could be addressed by simply increasing the administered dose has not been tested. Similarly, it is entirely possible that preferential effectiveness of sulfonylureas may only manifest itself early in the disease course, when beta cell function has not yet markedly deteriorated. As discussed further (below), the particular metabolic state of an individual may contribute additional variability to gene $\times$ drug interactions.

\section{Target identification: the elusive molecular target of metformin}

Metformin is the first-line agent in the treatment of type 2 diabetes [15-17]. Because of its effectiveness, low cost and favourable side effect profile, metformin is recommended as the first choice by all professional organisations (in the absence of contraindications) and is used in this manner by healthcare practitioners worldwide [18]. Nevertheless, hyperglycaemia eventually progresses in a substantial number of patients, necessitating the escalation of therapy [19]. Of particular concern are the number of young people who eventually fail metformin [20] and our inability to predict in whom metformin will be sufficient.

\section{Molecular mechanisms of metformin action}

Despite metformin being available for several decades and its widespread use, the precise manner by which metformin inhibits hepatic gluconeogenesis and improves insulin sensitivity has not been elucidated [21]. It has been shown to inhibit mitochondrial complex I $[22,23]$ and thereby activate AMP-activated protein kinase (AMPK) [24, 25], but it is not clear if such activation is dependent on a direct interaction between metformin and mitochondrial proteins or AMPK, or if it occurs via the mediation of other signalling molecules (Fig. 1). Recent loss-of-function experiments have shown that AMPK is not essential for metformin activity [26], indicating that much remains to be clarified about metformin's molecular interactions in the cell and its specific targets. A recent study implicates cAMP signalling as a major target of metformin, but this is also likely to be caused by indirect effects [27]. Such a gap in our knowledge has precluded the development of analogous therapies that might target the relevant pathways.

\section{The impact of genetic variants on metformin response}

The genetic parsing of metformin responders remains similarly underexplored $[3,28]$. A few candidate gene studies have attempted to identify genetic predictors of metformin response. 


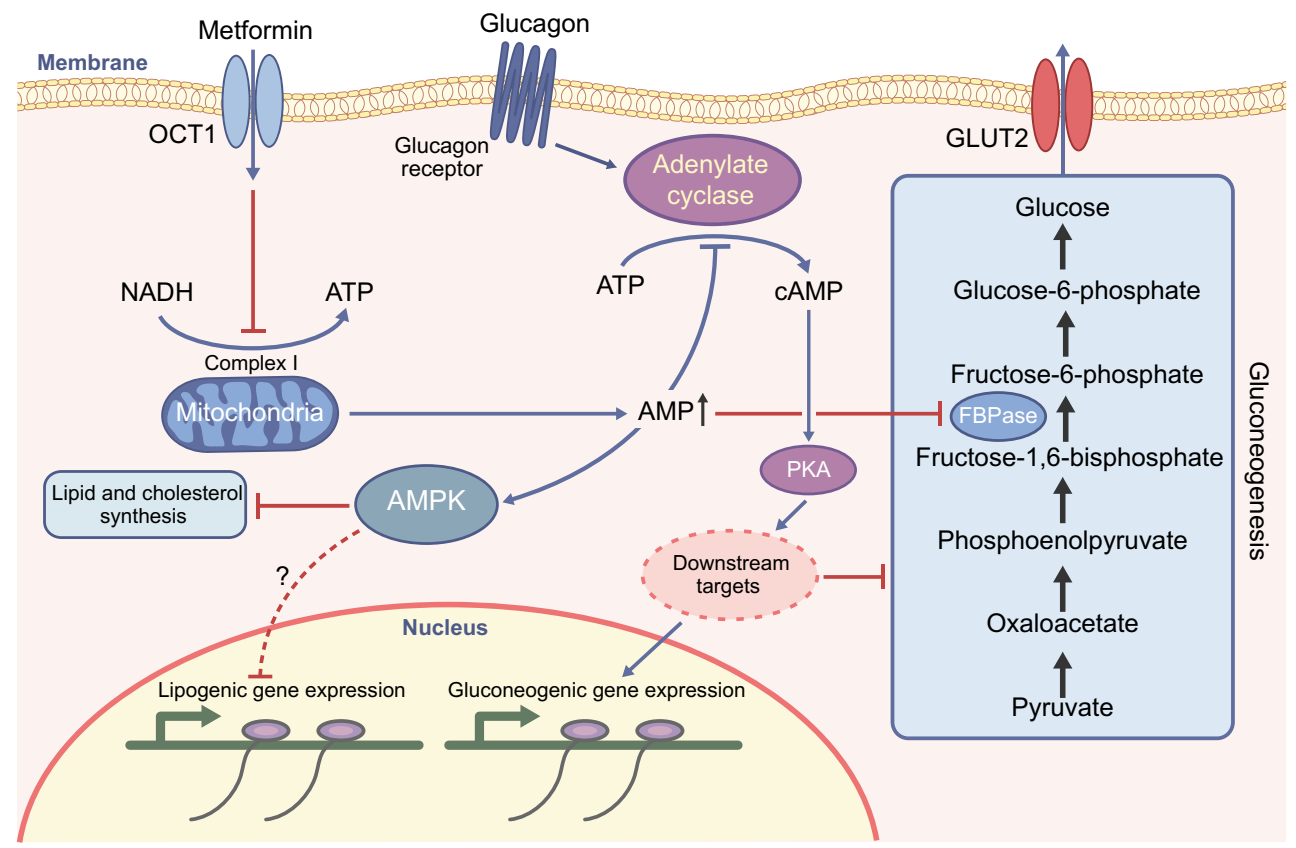

Fig. 1 Proposed molecular mechanisms of the blood-glucose lowering action of metformin. Metformin is transported into the hepatocyte by OCT1 and inhibits mitochondrial complex I via unknown mechanisms. As a result gluconeogenesis is impaired by: (1) decreased ATP and increased AMP (direct inhibition caused by the energy/ATP deficit); and (2) increased AMP, which interferes with cAMP-protein kinase A signalling, thus inhibiting fructose-1,6-bisphosphatase activity (a gluconeogenic enzyme). Increased AMP also leads to AMPK activation, thus reducing lipid/cholesterol synthesis and promoting beneficial long-term therapeutic effects. FBPase; fructose-1, 6-bisphosphatase; OCT1, organic cation transporter 1; PKA, protein kinase A. Figure redrawn from Rena et al [57], under the Creative Commons Attribution 4.0 International (CC BY) license (https://creativecommons.org/licenses/by/4.0)
SLC22A1 In a small cohort, missense variants in the organic cation transporter 1 (OCT1, encoded by SLC22A1) were associated with a reduced response to metformin [29]; subsequent follow-up in a large clinical retrospective cohort of patients with type 2 diabetes (the Genetics of Diabetes and Audit Research Tayside Study [GoDARTS]) failed to find support for such an association [30]. On the other hand, when combined with drugs that impair OCT1 function, loss-offunction variants at this locus increase the incidence of gastrointestinal side effects (Fig. 2) [31].

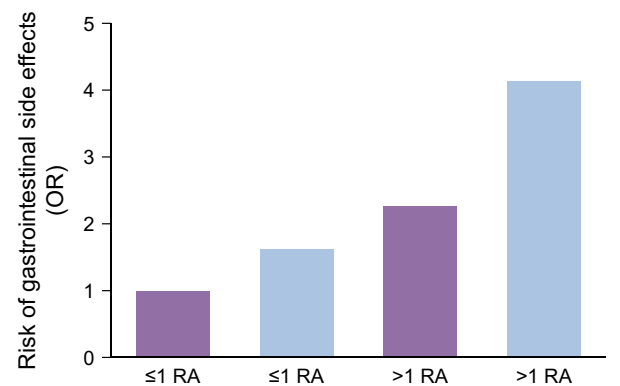

Fig. 2 When combined with drugs that interfere with OCT1 function, risk alleles (RA) at $O C T 1$ increase the incidence of gastrointestinal side effects. Such drugs include tricyclic antidepressants, citalopram, proton-pump inhibitors, non-dihydropyridine calcium channel blockers, doxazosin, spironolactone, clopidogrel, rosiglitazone, quinine, tramadol and codeine. Purple bars, without drugs; blue bars, with drug(s). Data obtained from [31]
SLC47A1 A pilot study in a small clinical retrospective cohort from Rotterdam (the Netherlands) identified a common polymorphism in the metformin transporter multidrug and toxin extrusion 1 (MATE1; encoded by SLC47A1, and responsible for disposing of metformin into bile and urine) as being associated with metformin response in patients with type 2 diabetes [32]. Accordingly, we found supportive evidence for a variant highly correlated with the index variant in the Diabetes

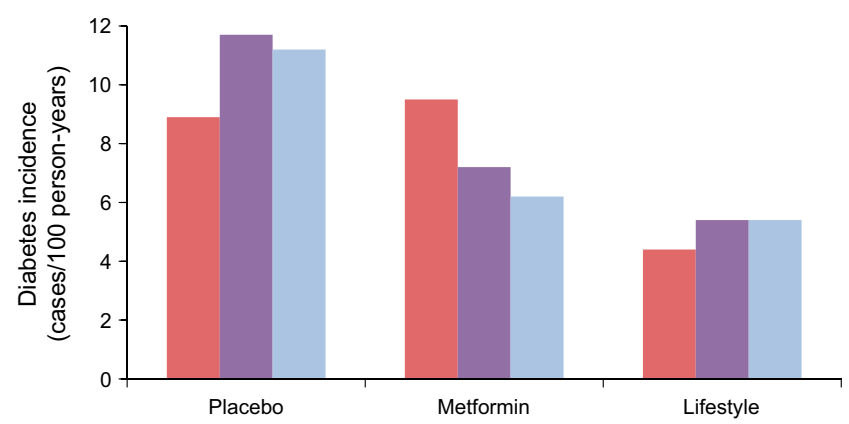

Fig. 3 Illustration of a gene $\times$ drug interaction. The genotype at SLC47A1 (SNP rs8065082) interacts with metformin in modulating diabetes incidence in DPP participants $\left(p_{\text {int }}=0.006\right)$. Genotypes: CC, red bars; CT, purple bars; TT, blue bars. Data obtained from [33]; American Diabetes Association (Diabetes, American Diabetes Association [2010]). Copyright and all rights reserved. Material from this publication has been used with the permission of American Diabetes Association 
Prevention Program (DPP), a randomised clinical trial that allocated participants at high risk of developing diabetes to placebo, lifestyle modification or metformin interventions (Fig. 3) [33]. This finding has been replicated in one small cohort [34] but not in a large-scale meta-analysis [35]. As has been the case for many other phenotypes, candidate gene studies have failed to produce a comprehensive picture of the genetic determinants of metformin action.

ATM Genome-wide association studies (GWAS) offer the opportunity to mine the entire genome for common variants associated with metformin response in an unbiased manner. In the first published GWAS for metformin response, the GoDARTS investigators analysed 705,125 SNPs in 1024 individuals in their retrospective clinical database. Fourteen SNPs in or near the gene encoding ataxia-telangiectasia mutated kinase $(A T M)$ were associated with categorical metformin response (defined as $\mathrm{HbA}_{1 \mathrm{c}}$ reaching $\leq 7 \%$ [ $\leq 53 \mathrm{mmol} / \mathrm{mol}]$ within 18 months of starting therapy) at $p<10^{-6}$. Consistent results were obtained for change in $\mathrm{HbA}_{1 \mathrm{c}}$ as a quantitative trait. Replication was attained for the top SNP (rs11212617) in 1783 additional GoDARTS participants and 1113 participants in the UK Prospective Diabetes Study (UKPDS) clinical trial [36], with joint analysis exceeding conventional genome-wide statistical significance $\left(p=3 \times 10^{-9}\right)$. Although this association has been replicated elsewhere [37], we have not found support for such an association in the DPP [38], using diabetes incidence or change in quantitative glycaemic traits as endpoints.

SLC2A2 Sample size is essential for genetic discovery. The Metformin Genetics (MetGen) Consortium has recently coalesced to assemble the largest resource of samples with DNA and information relevant to metformin response [39]; the majority of participants include patients with type 2 diabetes who have received metformin in the course of routine clinical care, and for whom glycaemic profiles are available in the form of serial $\mathrm{HbA}_{1 \mathrm{c}}$ measurements. In a recent report [40], the original genome-wide screen from the previous publication was extended to 1373 participants, with suggestive association signals undergoing three replication stages totalling nearly 14,000 participants. In this cohort, SNP rs8192675 in an intron of the gene $S L C 2 A 2$ (encoding the glucose transporter GLUT2) was associated with reduction in $\mathrm{HbA}_{1 \mathrm{c}}$ at genome-wide significance. This variant has both a primary effect on pre-metformin $\mathrm{HbA}_{1 \mathrm{c}}$ and an effect on posttreatment $\mathrm{HbA}_{1 \mathrm{c}}$ levels when adjusted for the baseline measurement. The same variant influences GLUT2 expression in the human liver, the tissue in which it is primarily expressed (though it is also present in beta cells) [41]. The genotype-driven difference approaches $0.33 \%$, with $0.50 \%$ typically used as a marker of glycaemic response that merits regulatory approval for a new drug. The effect translates to a dose difference of $550 \mathrm{mg}$ of metformin, again suggesting that perhaps differences in potency can be compensated by higher doses in the poorer responders. Similarly, we have not found evidence that this variant affects the ability of metformin to prevent diabetes in DPP participants with impaired glucose tolerance at baseline, indicating that perhaps genetic influences on drug response are also dependent on the metabolic state of the individual, with differential interactions occurring at diverse stages of disease progression. In this sense, pharmacogenetic findings for diabetes treatment may not overlap perfectly with those for diabetes prevention [42].

\section{Functional characterisation: $T C F 7 L 2$ and pharmacogenetics as a discovery tool}

The end-result of a GWAS, per se, is the identification of a genomic region harbouring a polymorphism in which a given allele is seen more frequently in disease than in health. If it is not clear how the polymorphism in question affects the expression or function of a nearby gene, the association signal may fail to point to a specific gene. Out of several possible candidate genes, one may be favoured if its pattern of expression is most consistent with the observed pharmacogenetic response (e.g. to an insulin secretagogue but not to an insulin sensitiser). Even if a gene is implicated via fine-mapping or expression studies, the mechanism by which changes in expression or function of its protein product cause type 2 diabetes is seldom obvious.

A case in point is TCF7L2. Discovered in 2006 as a gene containing a common intronic variant with a relatively strong effect on type 2 diabetes risk [43], it was not until 2010 that fine-mapping and functional studies conclusively proved that the rs7903146 variant was causal in influencing TCF7L2 expression in islets [44], although a recent report suggests that it may act via another effector transcript [45]. Physiological studies in the DPP first demonstrated that this type 2 diabetes-associated variant diminishes beta cell function [46], but the precise molecular mechanisms by which the protein encoded by TCF7L2 (transcription factor 7-like 2 [TCF7L2]) does so have proven elusive. Depending on the experimental model, TCF $7 L 2$ has been posited to influence beta cell proliferation early in development [47], proinsulin processing $[48,49]$, insulin vesicle docking [50] or incretin response [51, 52], although extra-pancreatic effects have also been suggested recently [53]. Given its proposed role in beta cell function, differential response to an insulin secretagogue might be expected in carriers vis-à-vis noncarriers of the type 2 diabetes risk allele: whether the drug 
improves response in carriers of the risk allele depends on its point of entry into the insulin secretion pathway relative to that of the gene. If the gene product acts downstream of the sulfonylurea receptor, for example, a sulfonylurea may still encounter the genetic block in inducing insulin secretion and carriers would show a worse clinical response; on the other hand, if the gene product acts upstream of the sulfonylurea receptor, a sulfonylurea drug may rescue the ability of the beta cell to secrete insulin and carriers would display a greater benefit.

The impact of sulfonylurea therapy by TCF7L2 genotype was retrospectively examined in the GoDARTS cohort. Treatment with sulfonylurea was less likely to achieve glycaemic goals in carriers of the TCF 2 L2 risk genotype, an effect not seen for metformin [54]. Similar effects were seen in a smaller cohort [55]. The tentative conclusion from these studies is that TCF7L2 impacts the insulin secretory pathway distal to the sulfonylurea receptor, as the drug seems less effective in risk allele carriers.

However, an alternative explanation could be considered. If variants in TCF7L2 reduce beta cell mass through either decreased proliferation or accelerated degeneration (leading to lower insulin secretion, a high proinsulin: insulin ratio and diminished efficacy of incretins), when used in patients with long-standing disease who have a reduced beta cell complement, sulfonylureas might be less effective; this difference would not be seen in non-diabetic individuals. We have conducted the Study to Understand the Genetics of the Acute Response to Metformin and Glipizide in Humans (SUGARMGH), a pharmacogenetic resource in which 1000 participants who were naive to antihyperglycaemic medications were given a single dose of glipizide followed by a short course of metformin, and glycaemic responses were measured in the acute setting (Fig. 4) [56]. In addition, incretin hormones were measured after an OGTT. This cohort replicates the known association of the T risk allele at TCF7L2 rs7903146 with fasting glucose, and can be used to ascertain whether the differentially worse response to sulfonylureas in $\mathrm{T}$ allele carriers with chronic type 2 diabetes observed by the GoDARTS investigators holds in the acute setting. Preliminary results suggest that this is not the case, again indicating that the metabolic state of the individual along the path of disease progression may modulate pharmacogenetic responses. On the other hand, indirect evidence confirms the potential role of incretin resistance in $\mathrm{T}$ allele carriers. Taken together, these findings do confirm that TCF $7 L 2$ exerts its effect on type 2 diabetes risk via its action on the beta cell, possibly by modulating incretin sensitivity (J.C. Florez, unpublished observations).

\section{Conclusions}

In pharmacogenetic studies, investigators should clarify a priori what the purpose of the research is, be it patient stratification, target identification or functional characterisation. Monogenic diabetes (neonatal diabetes and MODY) provides proof-of-concept that genetic information can guide therapy. Effect sizes in type 2 diabetes are much smaller but could be clinically meaningful, particularly if aggregated in genetic risk scores; whether this leads to a wider dosing range vs alternative drug choices needs to be tested. Furthermore, determinants of response may be contingent on the metabolic/ disease state of the individual or on their developmental stage. As an approach to discovery, agnostic genome-wide studies can help identify drug targets and modulators of drug response; additional benefits include the potential to assess likelihood of side effects and estimate heritability where family studies are unavailable or impractical. Given the small effects observed, whether genetic information improves clinical outcomes and is cost-effective needs to be studied: the strategy will only be viable if comprehensive genotyping for all clinically actionable variants is done prospectively by streamlining testing on a single, once-in-a-lifetime chip.

\section{SUGAR-MGH}

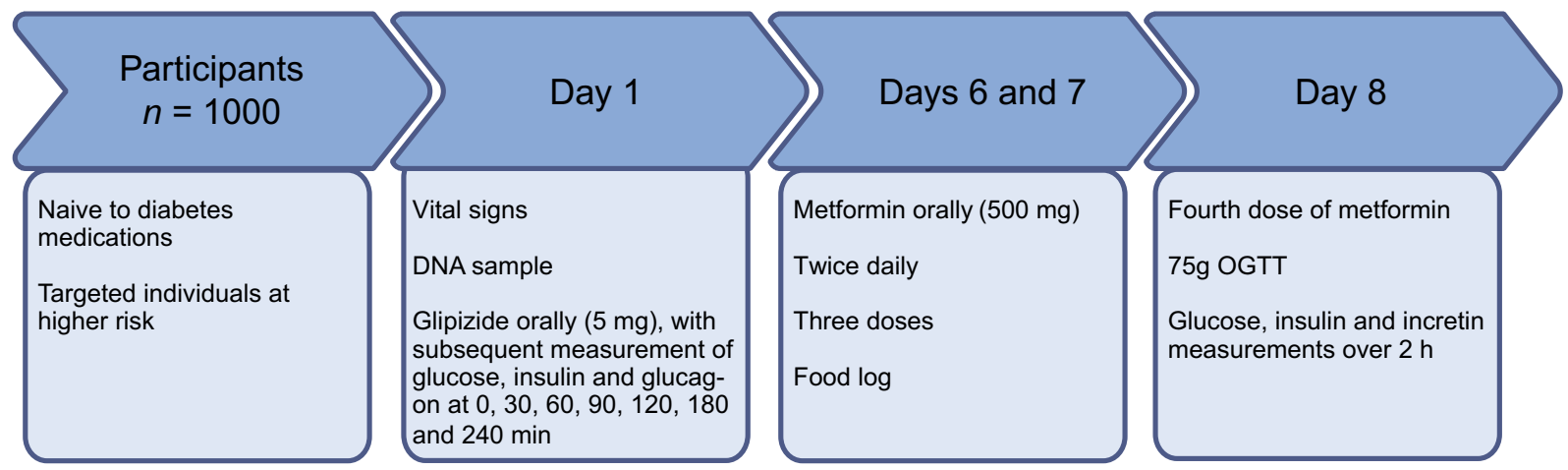

Fig. 4 Design of the Study to Understand the Genetics of the Acute Response to Metformin and Glipizide in Humans (SUGAR-MGH) [56] 
Funding JCF is supported by an unrestricted Massachusetts General Hospital Research Scholar Award. Parts of this work are supported by NIH/NIDDK R01 DK072041, NIH/NIDDK U01 DK105554, NIH/NIGMS R01 GM117163, NIH/NIDDK R01 DK105154, and NIH/NIDDK K24 DK110550.

Duality of interest JCF has received consulting honoraria from Merck and from Boehringer-Ingelheim.

Contribution statement JCF is the sole contributor to this paper.

\section{References}

1. Florez JC (2013) Pharmacogenetic perturbations in humans as a tool to generate mechanistic insight. Diabetes 62:3019-3021

2. Maruthur NM, Gribble MO, Bennett WL et al (2014) The pharmacogenetics of type 2 diabetes: a systematic review. Diabetes Care 37:876-886

3. Huang C, Florez JC (2011) Pharmacogenetics in type 2 diabetes: potential implications for clinical practice. Genome Med 3:76

4. Zhou K, Pedersen HK, Dawed AY, Pearson ER (2016) Pharmacogenomics in diabetes mellitus: insights into drug action and drug discovery. Nat Rev Endocrinol 12:337-346

5. Gloyn AL, Pearson ER, Antcliff JF et al (2004) Activating mutations in the gene encoding the ATP-sensitive potassium-channel subunit Kir6.2 and permanent neonatal diabetes. N Engl J Med 350:1838-1849

6. Babenko AP, Polak M, Cave $\mathrm{H}$ et al (2006) Activating mutations in the $A B C C 8$ gene in neonatal diabetes mellitus. N Engl J Med 355: 456-466

7. Greeley SA, Naylor RN, Philipson LH, Bell GI (2011) Neonatal diabetes: an expanding list of genes allows for improved diagnosis and treatment. Curr Diab Rep 11:519-532

8. Pearson ER, Flechtner I, Njolstad PR et al (2006) Switching from insulin to oral sulfonylureas in patients with diabetes due to Kir6.2 mutations. N Engl J Med 355:467-477

9. Gloyn AL, Weedon MN, Owen KR et al (2003) Large-scale association studies of variants in genes encoding the pancreatic $\beta$-cell KATP channel subunits Kir6.2 (KCNJ11) and SUR1 (ABCC8) confirm that the KCNJ11 E23K variant is associated with type 2 diabetes. Diabetes 52:568-572

10. Florez JC, Burtt N, de Bakker PI et al (2004) Haplotype structure and genotype-phenotype correlations of the sulfonylurea receptor and the islet ATP-sensitive potassium channel gene region. Diabetes 53:1360-1368

11. Hamming KS, Soliman D, Matemisz LC et al (2009) Coexpression of the type 2 diabetes susceptibility gene variants KCNJ11 E23K and $A B C C 8$ S1369A alter the ATP and sulfonylurea sensitivities of the ATP-sensitive $\mathrm{K}^{+}$channel. Diabetes 58:2419-2424

12. Feng Y, Mao G, Ren X et al (2008) Ser1369Ala variant in sulfonylurea receptor gene $A B C C 8$ is associated with antidiabetic efficacy of gliclazide in Chinese type 2 diabetic patients. Diabetes Care 31:1939-1944

13. Javorsky M, Klimcakova L, Schroner Z et al (2012) KCNJ11 gene E23K variant and therapeutic response to sulfonylureas. Eur $\mathrm{J}$ Intern Med 23:245-249

14. Ragia G, Tavridou A, Petridis I, Manolopoulos VG (2012) Association of KCNJ11 E23K gene polymorphism with hypoglycemia in sulfonylurea-treated type 2 diabetic patients. Diabetes Res Clin Pract 98:119-124

15. Inzucchi SE, Bergenstal RM, Buse JB et al (2015) Management of hyperglycaemia in type 2 diabetes, 2015: a patient-centred approach. Update to a position statement of the American Diabetes
Association and the European Association for the Study of Diabetes. Diabetologia 58:429-442

16. Nathan DM, Buse JB, Davidson MB et al (2009) Medical management of hyperglycemia in type 2 diabetes: a consensus algorithm for the initiation and adjustment of therapy: a consensus statement of the American Diabetes Association and the European Association for the Study of Diabetes. Diabetes Care 32:193-203

17. Rodbard HW, Jellinger PS, Davidson JA et al (2009) Statement by an American Association of Clinical Endocrinologists/American College of Endocrinology consensus panel on type 2 diabetes mellitus: an algorithm for glycemic control. Endocr Pract 15:540 559

18. Kirpichnikov D, McFarlane SI, Sowers JR (2002) Metformin: an update. Ann Intern Med 137:25-33

19. Kahn SE, Haffner SM, Heise MA et al (2006) Glycemic durability of rosiglitazone, metformin, or glyburide monotherapy. N Engl J Med 355:2427-2443

20. Zeitler P, Hirst K, Pyle L et al (2012) A clinical trial to maintain glycemic control in youth with type 2 diabetes. N Engl J Med 366: 2247-2256

21. Bailey CJ, Turner RC (1996) Metformin. N Engl J Med 334:574579

22. Owen MR, Doran E, Halestrap AP (2000) Evidence that metformin exerts its anti-diabetic effects through inhibition of complex 1 of the mitochondrial respiratory chain. Biochem J 348:607-614

23. El-Mir MY, Nogueira V, Fontaine E, Averet N, Rigoulet M, Leverve X (2000) Dimethylbiguanide inhibits cell respiration via an indirect effect targeted on the respiratory chain complex I. J Biol Chem 275:223-228

24. Fryer LGD, Parbu-Patel A, Carling D (2002) The anti-diabetic drugs rosiglitazone and metformin stimulate AMP-activated protein kinase through distinct signaling pathways. J Biol Chem 277: 25226-25232

25. Zhou G, Myers R, Li Y et al (2001) Role of AMP-activated protein kinase in mechanism of metformin action. J Clin Invest 108:11671174

26. Foretz M, Hebrard S, Leclerc J et al (2010) Metformin inhibits hepatic gluconeogenesis in mice independently of the LKB1/AMPK pathway via a decrease in hepatic energy state. J Clin Invest 120:2355-2369

27. Miller RA, Chu Q, Xie J, Foretz M, Viollet B, Birnbaum MJ (2013) Biguanides suppress hepatic glucagon signalling by decreasing production of cyclic AMP. Nature 494:256-260

28. Todd JN, Florez JC (2014) An update on the pharmacogenomics of metformin: progress, problems and potential. Pharmacogenomics 15:529-539

29. Shu Y, Sheardown SA, Brown C et al (2007) Effect of genetic variation in the organic cation transporter 1 (OCT1) on metformin action. J Clin Invest 117:1422-1431

30. Zhou K, Donnelly LA, Kimber CH et al (2009) Reduced function SLC22A1 polymorphisms encoding Organic Cation Transporter 1 (OCT1) and glycaemic response to metformin: A Go-DARTS study. Diabetes 58:1434-1439

31. Dujic T, Zhou K, Donnelly LA, Tavendale R, Palmer CN, Pearson ER (2015) Association of organic cation transporter 1 with intolerance to metformin in type 2 diabetes: A GoDARTS Study. Diabetes 64:1786-1793

32. Becker ML, Visser LE, van Schaik RHN, Hofman A, Uitterlinden AG, Stricker BHC (2009) Genetic variation in the multidrug and toxin extrusion 1 transporter protein influences the glucoselowering effect of metformin in patients with diabetes: A preliminary study. Diabetes 58:745-749

33. Jablonski KA, McAteer JB, de Bakker PI et al (2010) Common variants in 40 genes assessed for diabetes incidence and response to metformin and lifestyle intervention in the Diabetes Prevention Program. Diabetes 59:2672-2681 
34. Tkac I, Klimcakova L, Javorsky M et al (2013) Pharmacogenomic association between a variant in SLC47A1 gene and therapeutic response to metformin in type 2 diabetes. Diabetes Obes Metab $15: 189-191$

35. Dujic T, Zhou K, Yee SW et al (2016) Variants in pharmacokinetic transporters and glycaemic response to metformin: a MetGen metaanalysis. Clin Pharmacol Ther. doi:10.1002/cpt.567

36. Zhou K, Bellenguez C, Spencer CC et al (2011) Common variants near ATM are associated with glycemic response to metformin in type 2 diabetes. Nat Genet 43:117-120

37. van Leeuwen N, Nijpels G, Becker ML et al (2012) A gene variant near ATM is significantly associated with metformin treatment response in type 2 diabetes: a replication and meta-analysis of five cohorts. Diabetologia 55:1971-1977

38. Florez JC, Jablonski KA, Taylor A et al (2012) The $\mathrm{C}$ allele of ATM rs11212617 does not associate with metformin response in the Diabetes Prevention Program. Diabetes Care 35:1864-1867

39. Pawlyk AC, Giacomini KM, McKeon C, Shuldiner AR, Florez JC (2014) Metformin pharmacogenomics: current status and future directions. Diabetes 63:2590-2599

40. Zhou K, Yee SW, Seiser EL et al (2016) Variation in the glucose transporter gene $S L C 2 A 2$ is associated with glycemic response to metformin. Nat Genet 48:1055-1059

41. McCulloch LJ, van de Bunt M, Braun M, Frayn KN, Clark A, Gloyn AL (2011) GLUT2 (SLC2A2) is not the principal glucose transporter in human pancreatic beta cells: implications for understanding genetic association signals at this locus. Mol Genet Metab 104:648-653

42. Florez JC (2016) Leveraging genetics to advance type 2 diabetes prevention. PLoS Med 13:e1002102

43. Grant SFA, Thorleifsson G, Reynisdottir I et al (2006) Variant of transcription factor 7-like 2 (TCF7L2) gene confers risk of type 2 diabetes. Nat Genet 38:320-323

44. Gaulton KJ, Nammo T, Pasquali L et al (2010) A map of open chromatin in human pancreatic islets. Nat Genet 42:255-259

45. Xia Q, Chesi A, Manduchi E et al (2016) The type 2 diabetes presumed causal variant within TCF7L2 resides in an element that controls the expression of ACSL5. Diabetologia 59:2360-2368
46. Florez JC, Jablonski KA, Bayley N et al (2006) TCF7L2 polymorphisms and progression to diabetes in the Diabetes Prevention Program. N Engl J Med 355:241-250

47. Liu Z, Habener JF (2008) Glucagon-like peptide-1 activation of TCF7L2-dependent Wnt signaling enhances pancreatic beta cell proliferation. J Biol Chem 283:8723-8735

48. Loos RJF, Franks PW, Francis RW et al (2007) TCF7L2 polymorphisms modulate proinsulin levels and $\beta$-cell function in a British Europid population. Diabetes 56:1943-1947

49. Kirchhoff K, Machicao F, Haupt A et al (2008) Polymorphisms in the TCF7L2, CDKAL1 and SLC30A8 genes are associated with impaired proinsulin conversion. Diabetologia 51:597-601

50. da Silva Xavier G, Loder MK, McDonald A et al (2009) TCF7L2 regulates late events in insulin secretion from pancreatic islet betacells. Diabetes 58:894-905

51. Pilgaard K, Jensen CB, Schou JH et al (2009) The T allele of rs7903146 TCF7L2 is associated with impaired insulinotropic action of incretin hormones, reduced $24 \mathrm{~h}$ profiles of plasma insulin and glucagon, and increased hepatic glucose production in young healthy men. Diabetologia 52:1298-1307

52. Villareal DT, Robertson H, Bell GI et al (2010) TCF7L2 variant rs7903146 affects the risk of type 2 diabetes by modulating incretin action. Diabetes 59:479-485

53. Bailey KA, Savic D, Zielinski M et al (2015) Evidence of nonpancreatic beta cell-dependent roles of Tcf712 in the regulation of glucose metabolism in mice. Hum Mol Genet 24:1646-1654

54. Pearson ER, Donnelly LA, Kimber C et al (2007) Variation in TCF7L2 influences therapeutic response to sulfonylureas: A GoDARTs study. Diabetes 56:2178-2182

55. Schroner Z, Javorsky M, Tkacova R et al (2011) Effect of sulphonylurea treatment on glycaemic control is related to TCF7L2 genotype in patients with type 2 diabetes. Diabetes Obes Metab 13:89-91

56. Walford GA, Colomo N, Todd JN et al (2015) The study to understand the genetics of the acute response to metformin and glipizide in humans (SUGAR-MGH): design of a pharmacogenetic resource for type 2 diabetes. PLoS ONE 10:e0121553

57. Rena G, Pearson ER, Sakamoto K (2013) Molecular mechanism of action of metformin: old or new insights? Diabetologia 56:18981906 